\title{
ORDER IN AFFINE AND PROJECTIVE GEOMETRY
}

\section{Joe Lipman}

(received July 2, 1962)

In this note we give a characterisation of ordered skew fields by certain properties of the negative elements.

Properties of negative elements of a skew field $K$ a re then interpreted as statements about "betweenness" in any affine geometry over $\mathrm{K}$, or as statements about "separation" in any projective geometry over $\mathrm{K}$.

In this way, our axioms for ordered fields permit of immediate translation into postulates of order for affine or projective geometry (over a field). The postulates so obtained seem simple enough to be of interest. (cf. [4], p. 22)

1. We assume we have an ordered skew field $K$, [1], i. e. there is given a subset $P$ of $K$ (the positive elements) satisfying
a) $\mathrm{K}$ is the disjoint union of $\mathrm{P},(0),-\mathrm{P}$.
b) $P+P \subset P$.
c) $\mathrm{PP} \subset \mathrm{P}$.

Let $\mathrm{N}$ be the set of negative elements, i.e.

$$
N=\{\lambda \mid \lambda \notin P, \quad \lambda \neq 0\}
$$

so that

d) $P=\{\lambda \mid \lambda \notin N, \lambda \neq 0\}$.

Canad. Math. Bull. vol. 6, no.1, January 1963. 
For convenience we write $"\{\lambda\} "$ to mean $\lambda \in N$.

It is clear by elementary properties of ordered fields that

(i) $\mathrm{N}$ is not empty

(ii) $\{\lambda\} \rightarrow \lambda \neq 0$

(iii) $\{\lambda\} \rightarrow(1-\lambda) \in P$

(iv) If $\{\lambda\}$ and $\mu \in P$, then $\left\{\mu \lambda^{-1}\right\}$.

What we want is the converse: given a subset $\mathrm{N}$ of any field $K$, define $P$ by d), write " $\{\lambda\} "$ to mean $\lambda \in N$, and show that (i), (ii), (iii), (iv), imply a), b), c); in other words, if $\mathrm{N}$ satisfies (i), (ii), (iii), (iv), show that $\mathrm{K}$ can be ordered in such a way that $\mathrm{N}$ is the set of negative elements.

2. The proof is in eight steps.

I) $1 \in P$. Otherwise $\{1\}$, which by (iii) gives $0 \in P$.

II) With $\mu=1$, (iv) now gives $\{\lambda\} \rightarrow\left\{\lambda^{-1}\right\}$

III) Suppose both $\{\lambda\}$ and $\{-\lambda\}$. By (iii) $(1+\lambda) \in P$ and so by (iv) $\left\{(1+\lambda) \lambda^{-1}\right\}$ i.e. $\left\{\lambda^{-1}+1\right\}$ whence by (iii) $-\lambda^{-1} \in \mathrm{P}$. However, by II) $\{-\lambda\} \rightarrow\left\{-\lambda^{-1}\right\}$ and we have a contradiction. With (ii), this gives $\{\lambda\} \rightarrow-\lambda \in P$

IV) Suppose $\{\lambda\}$ and $\{\mu\}$. By II) and III) respectively, $\left\{\mu^{-1}\right\}$ and $-\lambda \in P$. (iv) then gives $\{-\lambda \mu\}$, and by III) $\lambda \mu \in P$. Thus NNCP.

V) By(i), there is a $\lambda$ such that $\{\lambda\}$. By III) $-\lambda \in P$ and so (iv) gives $\{-1\}$.

VI) If $\mu \in P$, then (iv), with $\lambda=-1$, gives $\{-\mu\}$. With III) this means $\mu \in P \leftrightarrow-\mu \in N$, i.e. $N=-P$. Thus $K$ is the disjoint union of $P,(0),-P$. This is a). 
VII) By IV) and VI) we have $\mathrm{PP} \subset \mathrm{P}$. This is c).

VIII) Again let $\lambda \in P, \mu \in P$. By VI) $\{-\lambda\}$, and so by (iv), $\left\{-\mu \lambda^{-1}\right\}$. Then (iii) gives $1+\mu \lambda^{-1} \in P$ and so $\left(1+\mu \lambda^{-1}\right) \lambda=\lambda+\mu \in P$ because of VII $)$.

Thus $\underline{P}+\mathrm{P} \subset \mathrm{P}$. This is $\mathrm{b})$ and the proof is complete.

3. For the geometric applications, we use the following well known facts of projective geometry (in two or more dimensions) over any skew field [2].

G1) Any three d.c. (for "distinct collinea $r^{\prime \prime}$ ) points may be taken into any other three d. c. points by a sequence of (at most three) perspectivities.

(A perspectivity is understood here to be a "projection" from a point.)

G2) With any four collinear points $W, X, Y, Z$, where $X, Y, Z$ are distinct and $W \neq Z$, we may associate a scalar $\lambda[W X, Y Z]$, the cross ratio of $W, X, Y, Z$ (in that order), satisfying

a) $\lambda[\mathrm{WX}, \mathrm{YZ}]=0$ if and only if $\mathrm{W}=\mathrm{Y}$.

b) For any scalar $\mu$ and any three d.c. points $X, Y, Z$, there is a unique point $W(\neq Z)$ such that $\lambda[W X, Y Z]=\mu$.

c) If the collinear points $\mathrm{W}, \mathrm{X}, \mathrm{Y}, \mathrm{Z}$ (X, Y, Z distinct, $W \neq Z)$ are taken into $W^{\prime}, X^{\prime}, Y^{\prime}, Z^{\prime}$ by a perspectivity, then $\left(X^{\prime}, Y^{\prime}, Z^{\prime}\right.$, are distinct, $W^{\prime} \neq Z^{\prime}$, and) $\lambda\left[W^{\prime} X^{\prime}, Y^{\prime} Z^{\prime}\right]=$ $\lambda[\mathrm{WX}, \mathrm{Y} Z]$.

G 3) A converse also holds. Let $\lambda\left[W^{\prime} X^{\prime}, Y^{\prime} Z^{\prime}\right]=\lambda[W X, Y Z]$; we may map $X, Y, Z$ onto $X^{\prime}, Y^{\prime}, Z^{\prime}$ by a sequence of perspectivities; then G2c) and G2b) imply that the image of $W$ is $W^{\prime}$; therefore $W, X, Y, Z$ may be mapped onto $W^{\prime}, X^{\prime}, Y^{\prime}, Z^{\prime}$ by a sequence of perspectivities.

G 4:) $\quad \lambda[W Y ; X Z]=1-\lambda[W X, Y Z]$ 
G 5) $\lambda[V W, Y Z]=\lambda[V X, Y Z](\lambda[W X, Y Z])^{-1}$

We consider now a projective space (always as sumed to be of two or more dimensions) over an ordered skew field $K$. As in section 1 , the set $N$ of negative elements of $K$ satisfies (i), (ii), (iii), (iv). Here again " $\{\lambda\}$ " shall mean $\lambda \in N$ (i. e. $\lambda<0$ ).

For any four d.c. points, W, X, Y, Z, (in that order) we define a relation $\langle W X, Y Z\rangle$ by

$$
<\mathrm{WX}, \mathrm{YZ}>\text { means }\{\lambda[\mathrm{WX}, \mathrm{YZ}]\} \text {. }
$$

"LWX, YZ>" is to be read "W, $\mathrm{X}$, separate $\mathrm{Y}, \mathrm{Z}$ ".

We do not define separation when any two of $W, X, Y, Z$ are equal, i. e.

S1) $\langle\mathrm{WX}, \mathrm{YZ}>\rightarrow \mathrm{W}, \mathrm{X}, \mathrm{Y}, \mathrm{Z}$ distinct.

In any projective geometry, the re exist three d.c. points. Thus condition (i) and $G 2 b$ ) give

S2) There exist four points $W, X, Y, Z$ such that $<W X, Y Z\rangle$.

Condition (iii) along with G4) gives

S 3) $<\mathrm{WX}, \mathrm{YZ}>\rightarrow$ not $<\mathrm{WY}, \mathrm{XZ}>$.

Condition (iv) along with G2a) and G5) gives

S4) $<\mathrm{WX}, \mathrm{YZ}>, \mathrm{V} \neq \mathrm{Y}, \mathrm{V} \neq \mathrm{Z}$, not $\langle\mathrm{VX}, \mathrm{YZ}\rangle$, togethe $\mathrm{r}$ imply $<\mathrm{VW}, \mathrm{YZ}>$.

Finally, G2c) gives

S5) The relation $\langle W X, Y Z\rangle$ is invariant under perspectivity.

When a projective geometry (over a skew field) has a relation on collinear points satisfying the separation axioms S1) - S5), we say that the geometry is ordered. 
4. The whole process is reversible.

Given an ordered geometry over a skew field $K$, we define a subset $\mathrm{N}$ of $\mathrm{K}$ by

$\lambda \in \mathrm{N}$ means that for some points $\mathrm{W}, \mathrm{X}, \mathrm{Y}, \mathrm{Z}$

$\lambda=\lambda[\mathrm{WX}, \mathrm{YZ}]$ and $\langle\mathrm{WX}, \mathrm{YZ}\rangle$

Remark: S5) and G3) then imply that if also $\lambda=\lambda\left[W^{\prime} X^{\prime}, Y^{\prime} Z^{\prime}\right]$ then $\left\langle W^{\prime} X^{\prime}, Y^{\prime} Z^{\prime}\right\rangle$.

Once more, we use the (somewhat overworked) notation " $\{\lambda\} "$ to signify $\lambda \in \mathrm{N}$.

It is then routine to verify that:

S1) and S2) imply condition (i).

G2a) and S1) give condition (ii).

G4), S3), G2a) and the above remark imply condition (iii).

G5), S4) and G2b) imply condition (iv).

Accordingly (section 2) we get an ordering on $K$ for which $\mathrm{N}$ is the set of negative elements. It is easily checked that the procedures of sections 3 and 4 are inverse to each other.

What has been established then, is the theorem that an ordering of a projective geometry ove $\mathrm{K}$ is equivalent to an ordering of $\mathrm{K}$.

The main steps involved in this statement a re reviewed in the following diagram.

$$
\mathrm{O}_{\mathrm{K}} \underset{\text { reference }[1]}{\stackrel{\text { reference }[1]}{\leftrightarrows}} \mathrm{P} \underset{\text { section 1 }}{\stackrel{\text { section 2 }}{\leftrightarrows}} \mathrm{N} \underset{\text { section 3 }}{\stackrel{\text { section 4 }}{\leftrightarrows}} O_{G}
$$


$\mathrm{O}_{\mathrm{K}}$ : order relation on skew field $\mathrm{K}$.

$P: \quad$ subset of $K$ satisfying a), b), c).

$N$ : subset of $K$ satisfying (i), (ii), (iii), (iv).

$\mathrm{O}_{\mathrm{G}}$ : order relation in a geometry over $\mathrm{K}$.

5. For affine geometry, we proceed in a similar way.

Instead of four d.c. points we now consider three. The associated scalar $\lambda[W X Y]$ is the ratio of the line segments (vectors) from $W$ to $X$ and from $Y$ to $X$ [3].

Instead of G1), we use the affine theorem that any two d. c. points may be mapped on any other two $3 . c$. points by a sequence of (at most three) parallel projections (perspectivities with center at infinity).

The relevant ternary relation $\langle W X Y\rangle$ (X lies between $W$ and $Y$ ) will satisfy

B 1) $\langle\mathrm{WXY}\rangle \rightarrow \mathrm{W}, \mathrm{X}, \mathrm{Y}$ distinct

B 2) There exist three points W, X, Y such that $\langle\mathrm{WXY}\rangle$

B 3) $\langle\mathrm{WXY}\rangle \rightarrow$ not $\langle\mathrm{WYX}\rangle$

B 4) $\langle\mathrm{WXY}\rangle, \quad \mathrm{V} \neq \mathrm{X}$, not $\langle\mathrm{VXY}\rangle$, together imply $\langle\mathrm{VXW}\rangle$

B 5) The relation is invariant under parallel projection.

The interested reader can fill in the details by analogy with sections 3 and 4 .

The final result is that an ordering of an affine geometry over a skew field $K$ is equivalent to an ordering of $K$.

Different approaches to this result can be found in

P. Scherk's paper in this is sue, and in [1] (Theorem 2.21). 


\section{REFERENCES}

1. E. Artin, Geometric Algebra, Interscience, 1957.

2. Veblen and Young, Projective Geometry, Vol. 1, Ginn and Co. , 1910.

3. , Vol. 2, 1918.

4. H. S. M. Coxeter, The Real Projective Plane, McGrawHill, 1949.

Summer Research Institute

Canadian Mathematical Congress 